Original Research Paper

\title{
Research of Effect of Peculiarities of Creasing a Polymer Sheet Material on a Rigid Base on Parameters of a Folding Operation
}

\author{
Il'dus Zakirov, Yuriy Kataev, Alexandr Nikitin and Nargiz Faizullina \\ Department of Strength of Structures, \\ Kazan National Research Technical University Named After A.N. Tupolev, Kazan, Russia
}

\author{
Article history \\ Received: 07-12-2016 \\ Revised: 19-05-2017 \\ Accepted: 19-08-2017 \\ Corresponding Author: \\ Nargiz Fayzullina \\ Department of Strength of \\ Structures, Kazan National \\ Research Technical University \\ Named After A.N. Tupolev, \\ Kazan, Russia \\ Email: nargizzzka@mail.ru
}

\begin{abstract}
Folded elements are widespread in design of various products. They are made of sheet metal, cardboard, plastics or other materials. For example, heat exchangers, printed products, cardboard or plastic folders, folded fillers for three- or multilayered panels, which are made of paper, cardboard or plastics material can be mentioned. Although folded fillers are significantly advanced in terms of structural or operational performance, their wide application is hindered by poor mastering of the mass production technology. At present, the process of shaping folded fillers using a creasing operation on a rigid base is the most prospective. However, the creasing operations on polymer materials while manufacturing folded fillers are performed with deformations significantly exceeding a uniform strain limit. There are no research works on the subject in the known technical literature. Therefore, the work intended to resolve the matters filling the gap is relevant. The purpose of the research is to obtain results enabling to develop optimal processes for shaping folded fillers of polymer materials taking into account considerations on the creasing on a rigid base. Based on the analysis of the initial and the final condition of the material at the creasing area, a mechanism of strain has been developed, objectives of determinations of the final shape in the creasing area have been solved analytically using an equilibrium equation adopted for the objectives in question; conditions for incompressibility, true (logarithmic) strain values, flat cross-section hypothesis have also been determined. As a result of the research, analytic dependences have been obtained for determinations of the residual thickness, curvature of the material at the creasing area, residual interfacial angle after release of the load. Experimental studies of the above parameters were carried out and comparisons with calculated values are shown.
\end{abstract}

Keywords: Creasing, Folding, Polymer Material, Creasing Depth, Residual Thickness, Bending Momentum

\section{Introduction}

A relatively large amount of papers is devoted to research of creasing and folding operations. They are mainly related to determination of parameters of these operations when using cardboard or corrugated cardboard material. The feature of these operations with cardboard is that the effect of multi-layered material is used. Due to the fact that segregation occurs during creasing, each layer works independently in bending and the final bending moment depends on the sum of bending moments of internal forces of each separate layer. To achieve a significant segregation of the material, the creasing should be performed using the following procedure: The creasing punch in the creasing area is penetrating a die cavity along with the work piece, then, folding to the opposite side of the creasing occurs and this is accompanied by bending of the stratified portion. The bending moment required for these operations will be significantly smaller than the bending moment of the initial material in the un segregated state. Thus, during the creasing of cardboard, the effect is 
achieved not by decreasing the thickness of the initial material but by stratification of separately-working layers during the folding. Beex and Peerling (2009) considered matters of cardboard segregation in the creasing area. This paper described the processes occurring in working zones of the folding and creasing. The paper showed that the experimental data confirmed the developed model and quite reliably predicts the behavior of the material. Nagasawa et al. (2003) considered the effect of the creasing punch stroke on the value of the bending moment for the cardboard. The authors have analyzed deformation angles, change of bending moment and its hysteresis factor according to the shear deformation (movement distance of the creasing punch). The ratio between hysteresis areas and the parameters of bending moment was determined.

However, as the deformation of the cardboard is different from isotropicelasto plasticity of ductile metals and working features are complex, papers on creasing have been based on the empirical expertise. Several accounts are known for suitable or critical working conditions. Planar elongation of the cardboard was considered by Halladay and Ulm (1939).

Several known test methods, such as ultimate tensile and bending strength, were compared by Donaldson (1954) to the quality in a creased sample of cardboard. Additionally, the actual creasing range was researched by Hine (1959) as a ratio of the creasing depth and width. Nevertheless, there are almost no considerations of the mechanism of stratification of paper fibers and changes of such mechanical properties as the bending rigidity in earlier researches.

Theoretical bases for describing disruption of the cardboard were stated by Xia in the paper. The proposed model consisted to two parts: A flat model and a surface model.

In the work by Nygårds et al. (2005), a five-layer cardboard was used to illustrate the work of the model. Anisotropic elastpoplastic properties of each layer of the cardboard were experimentally determined in terms of the uniaxial extension and compression, while traversal properties of the layers were determined by means of uniaxial tests for extension, compression and shear. Features of the material necessary for the model to characterize layers of the cardboard were stated in the paper by Xia (2002), same as the calibration of parameters. Experimental data were measured and presented by Stenberg (2002). Computational models for the creasing and folding were proposed by Xia and Choi.

The mechanical model by Choi and his colleagues used the description of the material proposed earlier by Mäkelä and Östlund (2003) that combines the model by Ramberg-Osgud and the model by Karafillis and Boyce (1993). Choi expanded the description with a non-linear resilient behavior in $\mathrm{ZD}$. He checked the model during experiments with folding.
Researches by Stenberg (2003) offered material models of paper for through-thickness behavior, while Sawyer et al. (1998; Castro and Ostoja-Starzewski, 2003) proposed the models for the planar behavior.

Description of the paper deformation process was presented in papers by Isaksson et al. (2004) Moreover, Isaksson and Hägglund (2005) use the energy release rate at the top of a crack as a disrupting criterion for stratification. Beldie et al. (2001; Barbier et al., 2004; Thakkar et al., 2008) used the usual deformability to model the behavior of the cardboard and corrugated cardboard.

In the work by Zakirov et al. (2015), the possibility of creating a relatively high corrugation core using creasing and folding operations is considered. An important issue in this respect is obtaining a flat pad as one of the elements of the corrugation at points of its contact with the outer panels. Two schemes of folding of preliminary creased cardboard sheet were studied. The potential of both these schemes was evaluated. Shape and design of the creasing punch that provides quality contact pads within a wide range of sizes of corrugations were suggested.

However, the information about creasing and folding of polymer materials is rather limited. Experimental studies of creasing Nomex and Kevlar polymer papers were published by Zakirov and Zakirov (2014). The paper presents theoretical dependences of a combined operation of creasing and folding on an elastic base, of the residual force of bending angle, of the residual thickness in the creasing zone, on penetration depth of the work piece with the punch into the elastic base. The article by Zakirov et al. (2016) describes force factors of the creasing process on the elastic basis too. Analytical dependences for determination of the force of creasing and folding and the value of the residual angle after the spring action were also shown in the paper.

The creasing and folding of a one-layer polymer material differs due to the fact that the effect of folding in these operations is obtained by thickness reduction of the initial material. If bending moment versus thickness relation is known, then it can be calculated that a 2-times thickness decrease causes an 8-times decrease for the required bending moment. Therefore, it is important to know the actual value of the thickness change for the material in the creasing zone after a certain displacement of the creasing punch.

Industrially, the creasing of the polymer material on the rigid base is more prospective. According to experiments, a dimple (a groove or a channel, etc.) appears on the other side of the work piece (on the side opposite the creasing punch) during the creasing process on a hard base. This occurs because of the stressed state of the sample under load.

Figure 1 illustrates the work piece section in the creasing zone. 


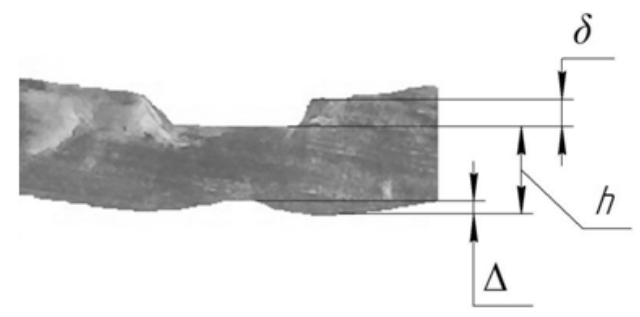

Fig. 1 Work piece section in the creasing zone

The described feature of the creasing operation on a polymer material during the determination of the material parameters has not been considered before.

The aim of this study is to obtain results enabling to develop optimal shaping processes for folded fillers made of polymer materials. This requires that the problem of identification of the dependence of the dimple depth versus the operation parameters of the creasing should be solved, that the identified dependences should be checked experimentally and the estimated values of the bending moment at a residual thickness considering the depth of the dimple should be checked experimentally as well as.

\section{Proposed Methodology}

\section{Analytic Definition of a Residual Thickness in the Creasing Area}

For the solution of the problem regarding the analytical determination of the creasing parameters on a rigid base, equilibrium equations were used adopted to the problem in question, as well as elasticity conditions, true (logarithmic) deformations, flat cross-section hypothesis.

Assuming incompressibility of the material in absence of friction forces, the deformation of tensile strain of a sheet can be determined from the following condition (Fig. 2): $d y=(H-y) d x$.

After rearrangement:

$$
\int_{0}^{\delta} \frac{a d y}{H-y}=\int_{0}^{\varepsilon x} d x
$$

After integration:

$$
\varepsilon x=\operatorname{In} H / h
$$

The tension deformation of the sheet remains constant throughout the sheet thickness.

During creasing, there is a friction between surfaces of the creasing punch, the sheet material and the base. The above leads to a variable deformation of strain over the thickness $h$ below the surface of the creasing punch during introduction of the creasing knife to the depth of $\delta$.
Frictional forces between the creasing punch and the sheet material hinders a movement of the sheet material in the direction away the axis of symmetry of the creasing punch. A complete absence of such movements can occur under certain conditions. Deformations will increasingly occur in a part of the sheet which is remote from the surface of the punch. Taking into account the flat cross-section hypothesis, it can be assumed that the strain will change linearly over the thickness of the sheet.

Let us consider the state of stress of the sheet under the creasing knife. For making an equation we will select an infinitely small element of height with a thickness $d x$ and a height $h$ and consider its equilibrium condition (Fig. 3):

$\left(\sigma_{x}+\frac{\partial \sigma_{x}}{\partial \sigma} d x\right) h-\sigma_{x} h-P\left(f_{1}+f_{2}\right) d x=0$

Where:

$\sigma_{x} \quad=$ Stress in the cross section of the sheet

$P \quad=$ Tool pressure on the sheet

$f_{1}$ and $f_{2}=$ Coefficients of friction between the creasing punch and the sheet, between the sheet and the rigid base, respectively

Assuming that the sheet material has an ideal plasticity, we can write the condition for the onset of the plastic condition:

$\sigma_{x}+P=\sigma_{T}$

From the known solutions (1) and (2), we get:

$d P h+\left(f_{1}+f_{2}\right) P d x=0$

Upon integrating:

$P=c e^{-\frac{\left(f_{1}+f_{2}\right)}{h} x}$

where, $\mathrm{c}$ is the integration constant.

The integration constant could be found from the following boundary condition: When $x=a$ : $\sigma_{x}=\sigma_{0}$, where $\sigma_{0}$ is a potential extra tension of the sheet considering the above, we find:

$P=\sigma^{*} e^{\frac{\left(f_{1}+f_{2}\right)}{h}(a-x)} \sigma^{*}=\sigma_{T}+\sigma_{0}$

Let us define the value of the total force exerted on the sheet:

$$
P=2 \int_{0}^{a} \sigma^{*} e^{\frac{f_{1}+f_{2}}{h}(a-x)} d x
$$




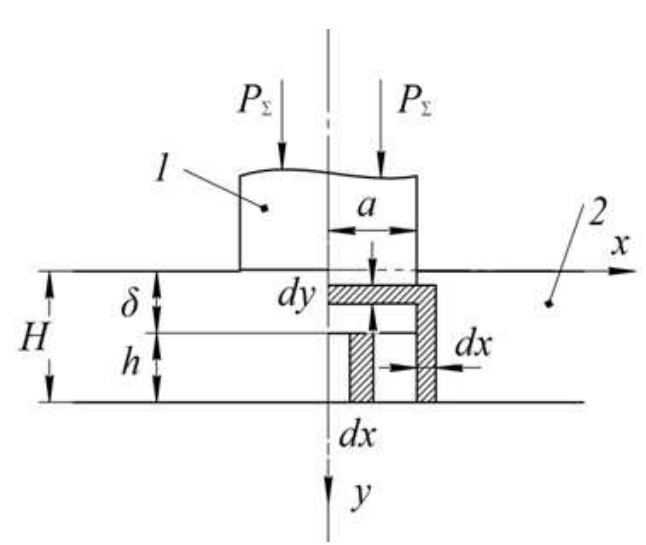

Fig. 2. The scheme of penetration of a creasing punch into sheet work piece 2

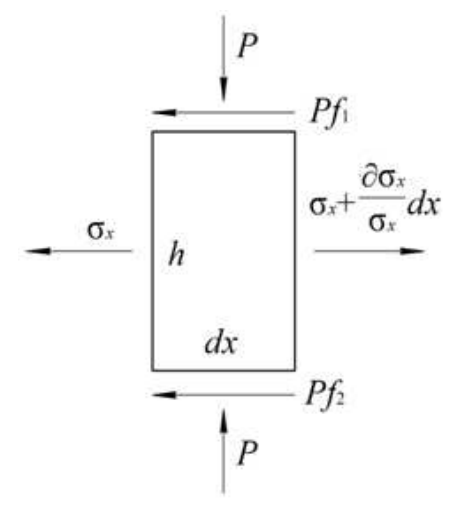

Fig. 3. Incrementally small element of the sheet

Upon integrating:

$P=\frac{2 \sigma^{*} h}{f_{1}+f_{2}}\left(e^{\frac{f_{1}+f_{2}}{h} a}-1\right)$

If friction forces are on one of surfaces of the sheet (Fig. $4 a$ and $b$ ), then the total force of creasing will be written as follows:

$P_{1}=\frac{2 \sigma^{*} h}{f_{1}}\left(e^{f_{1} \frac{a}{h}}-1\right), P_{2}=\frac{2 \sigma^{*} h}{f_{2}}\left(e^{f_{2} \frac{a}{h}}-1\right)$

where, $P_{1}$ is the pressure of creasing when friction occurs between the creasing knife and the sheet (Fig. 4a) and $P_{2}$ is the pressure of creasing when friction occurs between the sheet and the hard base (Fig. 4b).

Deformations $\varepsilon_{1}$ and $\varepsilon_{2}$ are caused by the friction coefficients $f_{1}$ and $f_{2}$ and can be represented with the ratio:

$$
\frac{\varepsilon_{1}}{\varepsilon_{2}}=\frac{P_{2}}{P_{1}}
$$

From the combined solution of (7) and (8) we find:

$$
\varepsilon_{1}=k \varepsilon_{2}, k=\frac{\left(e^{f_{2} \frac{a}{h}}-1\right) f_{1}}{\left(e^{f_{1} \frac{a}{h}}-1\right) f_{2}}
$$

On the basis of the condition of incompressibility, we have:

$\varepsilon_{1}+\varepsilon_{2}=2 \varepsilon_{x}$

From the ratio of (9) and (10), we get:

$\varepsilon_{2}=\frac{2 \varepsilon_{x}}{1+k}$

An uneven deformation over the thickness $\mathrm{h}$ leads to a bending of the sheet (Fig. 5).

The parameters of the bended sheet are defended with deformations $\varepsilon_{1}$ and $\varepsilon_{2}$ :

$h^{*}=\frac{\varepsilon_{2}}{\varepsilon_{2}-\varepsilon_{1}} h, \varkappa=\frac{\varepsilon_{2}-\varepsilon_{1}}{h}, \varkappa=\frac{1}{\rho}$

where, $\rho$-radius of curvature of the conditional sheet layer which is not exposed to tensile strain deformation, $h^{*}$ - coordinate determining the position of the layer.

Radius of curvature $\rho_{H}$ of the outer layer of the sheet will be:

$\rho_{H}=\frac{h\left(1+\varepsilon_{2}\right)}{\varepsilon_{2}-\varepsilon_{1}}$

The $\Delta$ parameter, in accordance with Fig. 5, is determined as:

$\Delta=\rho_{H}-\rho_{H} \cos \alpha$, where $\alpha=\frac{a \varepsilon_{2}}{\rho_{H}}$

After removal of the knife from the surface of the work piece, a straightening of the surface of 2-6 will occur under the influence of internal stresses of compression. Point 5 goes to position 5', point 4 goes to position 4 ', which will lead to a displacement of point 2 to $2^{\prime}$ (Fig. 6).

As a result, after the thin-walled sheet creasing operation on the outer surface, a crease groove with depth $c$ is formed on the opposite side of the sheet. Considering that $c$ equals $\Delta$ (Fig. 5) and using functions (13) and (14), the following equation can be written as:

$c=\frac{h\left(1+\varepsilon_{2}\right)}{\varepsilon_{2}-\varepsilon_{1}}(1-\cos \alpha)$ 


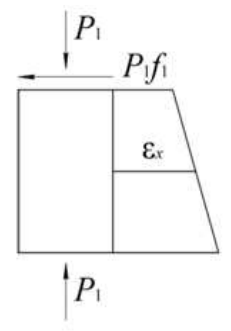

(a)

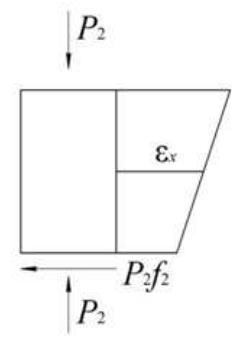

(b)

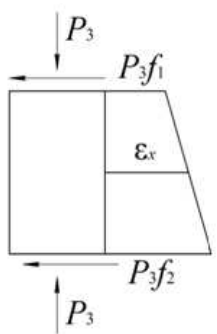

(c)

Fig. 4. Strain diagrams of the sheet: (a) -Friction between the creasing knife and the sheet, (b) -Friction between the sheet and the hard base, (c) -friction at both sides of the sheet

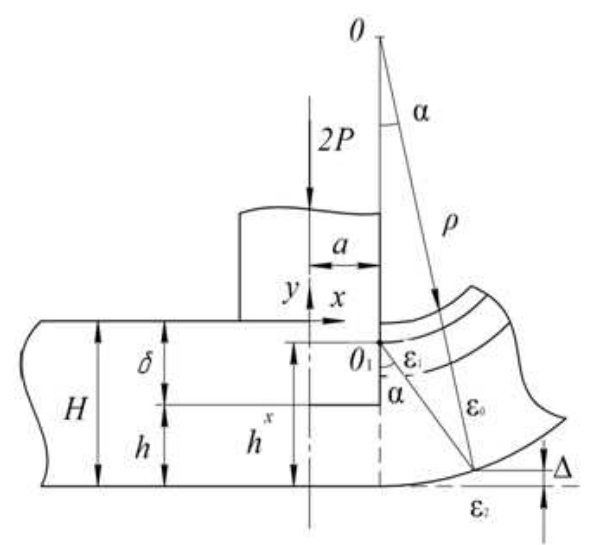

Fig. 5. The parameters of bending of the sheet being creased

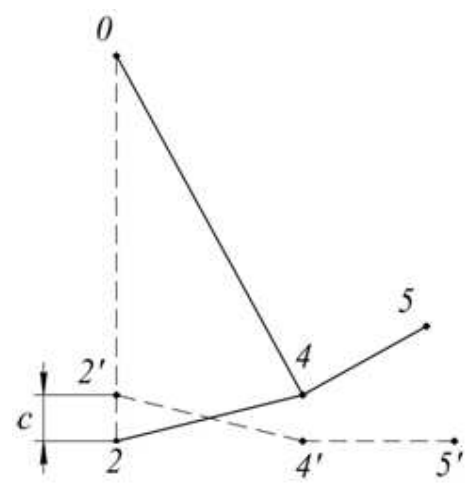

Fig. 6. The scheme of $2^{\prime}-3$ 'crease formation on the opposite side opposite to the creasing knife. Solid line is the position of the outer surface of the sheet after the penetration of the creasing knife, dashed line is the position of the outer surface after removal of the creasing knife

Calculated graphs $c=f(H, \delta, a)$ in accordance to the formula (15) with $H=1.0$; and width of the creasing knife is $1 \mathrm{~mm}$ in the range of penetration depth of $\delta / H$ 0,2-0,5 are shown in Fig. 9.

\section{Determination of Folding Bending Moment}

The external bending moment is determined at the condition of its equilibrium with the moment of internal forces. According to recommendations stated in (Donaldson, 1954), moment of internal forces of bending on a radius lesser than 5 thicknesses can be determined as:

$M=1.5 W \sigma_{6} n$

Where:

$W=$ Resisting moment (for a rectangular cross-section $W=b * S 2 / 6$ )

$n=1,8$ is a factor characterizing the effect of hardening

$\sigma_{b}=$ The tensile strength of the material

The formula (16) shows that the material thickness has a crucial influence on bending moment. In our case, the thickness is $S=H-\delta-c$.

\section{Experimental Study}

\section{Experimental Studies of Creasing and Folding}

Experimental studies were conducted on PVC material (GOST 9998-86 - Polyvinylchloride films for household use) at the relative air humidity of $(50 \pm 5)$ $\%$ and the ambient temperature of $(23 \pm 2)^{\circ} \mathrm{C}$. Material characteristics: Thickness is $1.0 \mathrm{~mm}$, maximal uniform strain at tension is 0.15 ; modulus is $0.597 \mathrm{GPa}$. Sample size is $30 \times 40 \mathrm{~mm}$. The creasing punch thickness $(2 a)$ is $1 \mathrm{~mm}$. Displacement depths of the creasing punch $(\delta)$ were $0.2,0.3,0.4$ and $0.5 \mathrm{~mm}$. Displacement depths of the creasing knife were checked with a dial indicator with the accuracy of $0.01 \mathrm{~mm}$. The Fig. 7 shows the arrangement of the creasing experimental installation.

The experimental installation contains a movable rod 1 with a socket for attachment of a creasing punch 2 on the lower plate of rack 3 , a rigid base 4 is installed. A work piece 5 is placed on the rigid base 4 to monitor the working displacement of the creasing punch 2 , a dial indicator 6 is fixed on the movable rod 1 .

Three samples were used for each penetration depth. Photographing and measurement of the creasing depth were carried out on the apparatus shown on Fig. 8. 


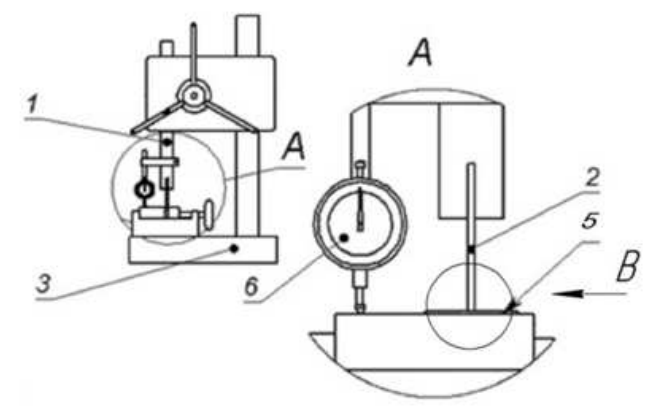

(a)

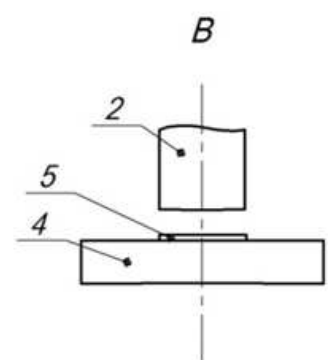

(b)

Fig. 7. Creasing experimental installation: (a) arrangement of the creasing experimental installation; (b) a device for the material creasing

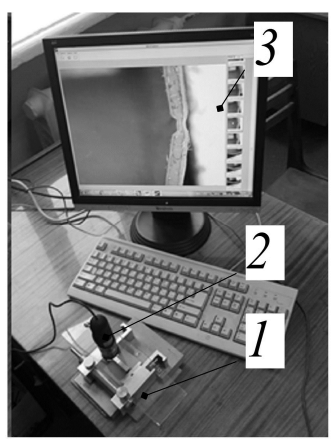

Fig. 8. The apparatus for measurement of the creasing depth

The apparatus contains unit 1 , which is used for fixation of the sample based at a side edge and includes a digital microscope 2, whereby the sample is photographed. The images are shown on a computer screen 3, where the measured parameters in question are recorded. Results of the measurement are shown in Table 1.

Graphs were plotted using the measurements done (Fig. 9), where calculated values are shown too.

A comparison of the calculated and the experimental values shows that in the range of operating residual thickness, a difference does not exceed $15 \%$. However, at low penetration depths of the creasing punch, values of $\delta$ and $\Delta$ are very small, for which reason a correction factor $\kappa$ was introduced in calculation formula. At the set values of $f_{1}=0.05$ and $f_{2}=0.25: \kappa_{1}=9,4, \kappa_{2}=3,5, \kappa_{3}=$ 1,8 and $\kappa_{4}=1$ with the creasing punch penetration of 0.2 , $0.3,0.4$ and $0.5 \mathrm{~mm}$, respectively.

Some samples were folded across the creasing line in a straight direction (Fig. 10a) (to the side of the creasing from the creasing punch side), while other samples were folded in the reverse direction (Fig. $10 \mathrm{~b}$ ) (to the back side) till closing of edges $\left(B C^{\prime}\right.$ position). Samples were photographed with the microscope after removal of the load (position $B C^{\prime \prime}$ ), marked angles were also measured as follows: $\alpha_{n}$ for samples folded in the straight direction; $\alpha_{0}$ for samples folded in the reverse direction.

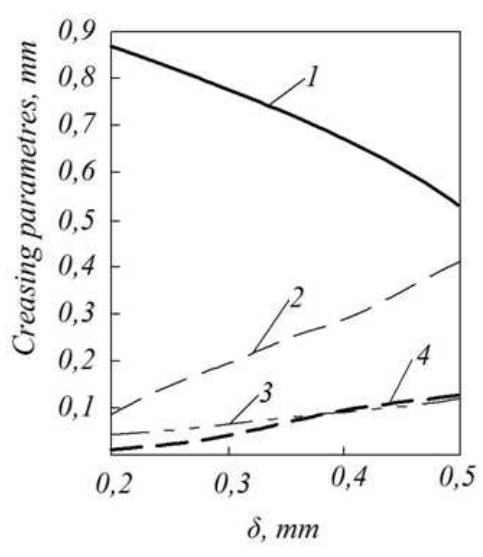

Fig. 9. A graphs illustrating dependences of the creasing parameters on the penetration depth of the creasing punch $(\delta)$ : 1 -residual thickness, 2 -creasing depth, 3 -dimple depth; 4 -calculated dimple depths

A measurement of sample sizes after the folding was performed with the apparatus shown in Fig. 8. The measurement values are shown in Table 2.

As the Table shows, folding the sample in the reverse direction after creasing on a rigid base makes the folding angle less by $12 \%$ than in case of folding in the straight direction and with increasing depth of penetration of the creasing punch, the difference is reduced. The Fig. 11 shows photos of the samples after creasing on the rigid base.

Figure 11 shows that after folding the sample in the reverse direction, there is an almost flat section at the creasing zone, while on the other hand, after folding in the straight direction, the outer area of the folding has a radius shape.

Experiments for determination of the bending moment for folding the samples were carried out. The folding of the samples was performed in a device installed in the testing machine Instron ${ }^{\circledR} 10000$ (Fig. 12). The folding time was $30 \mathrm{sec}$. The folding of the samples was conducted according to the straight and the reverse schemes. 


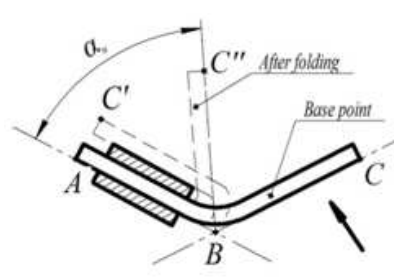

(a)

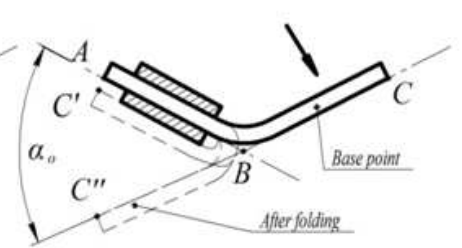

(b)

Fig. 10. The position of sample edges (a) for the straight folding scheme (b) for the reverse folding scheme

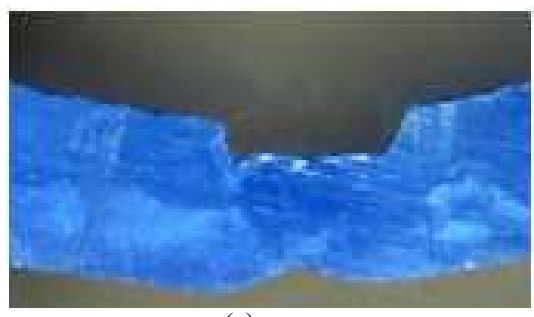

(a)

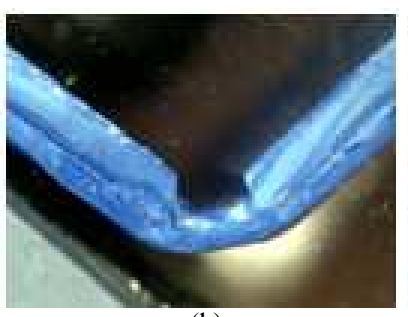

(b)

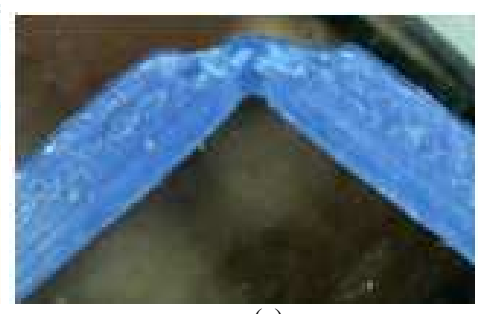

(c)

Fig. 11. Samples creased on a rigid base (a) -after creasing (b) -after folding in the straight direction (c) -after folding in the reverse direction

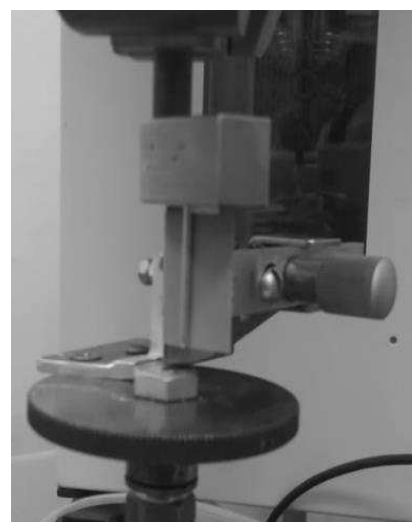

Fig. 12. Device for measurement of the bending moment for folding of the samples

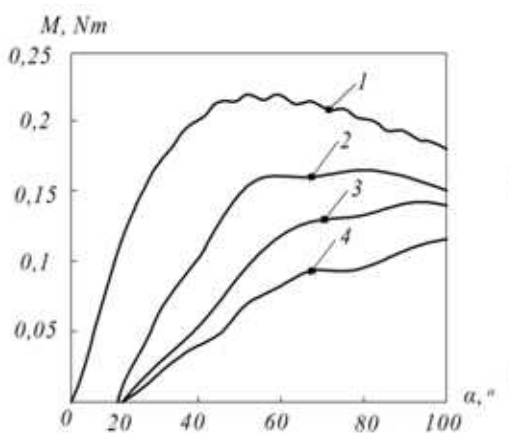

(a)

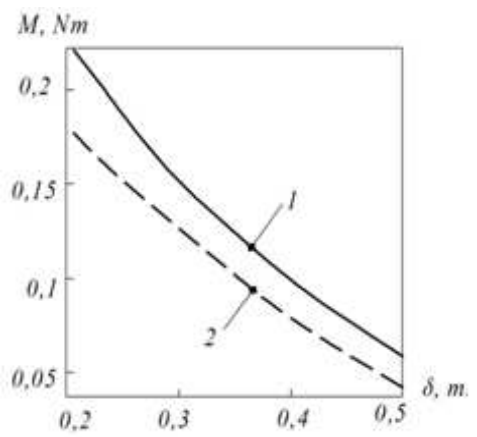

(b)

Fig. 13. (a) The graph of dependences of the bending moment on the punch penetration depth in $1 \mathrm{~mm}$ thick samples creased on a hard base, using the straight scheme of folding, where the knife thickness is $1 \mathrm{~mm}$ and the punch penetration depth is: (1) 0.2 $\mathrm{mm}$; (2) $0.3 \mathrm{~mm}$; (3) $0.4 \mathrm{~mm}$ and (4) $0.5 \mathrm{~mm}$; (b) the graph of the dependence of the bending moment on the penetration depth of the punch: (1) The experimental values, (2) the calculated values 
Table 1. Test values of creasing with the punch thickness of $1(\mathrm{~mm})$

\begin{tabular}{|c|c|c|c|c|}
\hline Sample no. & $\begin{array}{l}\text { Creasing depth on the } \\
\text { punch side }(\mathrm{mm})\end{array}$ & $\begin{array}{l}\text { Dimple depth on the } \\
\text { reverse side }(\mathrm{mm})\end{array}$ & $\begin{array}{l}\text { Residual thickness } \\
(\mathrm{mm})\end{array}$ & $\begin{array}{l}\text { Display readings } \\
(\mathrm{mm})\end{array}$ \\
\hline 1 & 0,09 & 0,04 & 0,89 & \\
\hline 2 & 0,09 & 0,03 & 0,86 & 0,2 \\
\hline 3 & 0,10 & 0,05 & 0,86 & \\
\hline Mean value & 0,09 & 0,04 & 0,87 & \\
\hline 4 & 0,21 & 0,06 & 0,77 & \\
\hline 5 & 0,18 & 0,06 & 0,75 & 0,3 \\
\hline 6 & 0,20 & 0,07 & 0,81 & \\
\hline Mean value & 0,20 & 0,06 & 0,78 & \\
\hline 7 & 0,29 & 0,08 & 0,65 & \\
\hline 8 & 0,28 & 0,11 & 0,70 & 0,4 \\
\hline 9 & 0,31 & 0,09 & 0,66 & \\
\hline Mean value & 0,29 & 0,09 & 0,67 & \\
\hline 10 & 0,42 & 0,14 & 0,54 & \\
\hline 11 & 0,40 & 0,12 & 0,53 & 0,5 \\
\hline 12 & 0,40 & 0,11 & 0,53 & \\
\hline Mean value & 0,41 & 0,12 & 0,53 & \\
\hline
\end{tabular}

Table 2. Sample sizes after the folding

\begin{tabular}{lllr}
\hline No. & Penetration depth $(\mathrm{mm})$ & Folding direction & Average angle, ${ }^{\circ}$ \\
\hline 1. & 0.2 & Straight & 137.82 \\
2. & 0.2 & Reverse & 89.48 \\
3. & 0.3 & Straight & 105.88 \\
4. & 0.3 & Reverse & 93.81 \\
5. & Straight & 96.65 \\
6. & Reverse & 94.90 \\
7. & 0.4 & Straight & 102.60 \\
8. & 0.5 & Reverse & 102.66 \\
\hline
\end{tabular}

The angle of the $B C$ edge in the initial position was assumed as $0^{\circ}$ angle (Fig. 10). Then the sample is folded in the direction shown with an arrow in Fig 10 and the edge $B C$ moves to $B C$ ' position. In this case, sample No.1 with the maximum angle of folding is taken as the initial position of the edge $B C$. Folding of next samples also starts from this position.

Figure 13 shows diagrams of dependences of the bending moment on the folding angle of the samples.

Figure 13 shows the experimental dependences of the bending moment value $M$ for folding the creased samples at different values of angle $\alpha$ of the creasing knife (punch) displacement, when the samples have been folded in the straight directions. The data analysis shows that in case of the reverse scheme of folding, the maximal value of the bending moment is averagely 20 $30 \%$ lower than in case of the straight scheme of folding.

\section{Conclusion}

In this study, the influence of features of the creasing of a polymer sheet on a hard base on the folding operation by means of measuring of the penetration depth of the punch and the determination of the residual thickness of the work piece in the creasing area is studied for in the subsequent folding is studied. The influence of the residual thickness on the bending moment is also considered. The following results have been obtained:

- The analysis of creasing formation operation on a polymer sheet material was performed. The mechanical model for the dimple formation on sheet material on side opposite the creasing punch was created on its basis. A mathematical model was developed and analytical dependences for determination of the depth of the dimple were obtained. A dependence of the dimple depth on the residual depth in the creasing area. It is about $20 \%$ of the creasing depth

- An analytical dependence for determination of the bending moment and the residual angle after folding and springing back was shown

- The parameters of a work piece in the area of creasing (creasing depth, dimple depth, residual thickness) were found experimentally

- Experiments were carried out to identify the effect of folding schemes on the value of the bending moment and the residual angle between the edges of the sample after folding. The difference is up to $15 \%$

\section{Acknowledgement}

The author thanks Kazan National Research Technical University for supporting this research. 


\section{Author's Contributions}

Il'dus Zakirov: Designed the research plan and organized the study.

Yuriy Kataev: Coordinated the data-analysis and contributed to the writing of the manuscript.

Alexandr Nikitin: Participated in all experiments, coordinated the data-analysis.

Nargiz Faizullina: Contributed to the writing of the manuscript and coordinated the mouse work.

\section{Ethics}

Authors should address any ethical issues that may arise after the publication of this manuscript.

\section{References}

Barbier, C., P.L. Larsson and S. Östlund, 2004. On dynamic effects at folding of coated papers. Composite Structures, 67: 395-402. DOI: 10.1016/j.compstruct.2005.01.003.

Beex, L.A.A. and R.N.J. Peerlings, 2009. An experimental and computational study of laminated paperboard creasing and folding. Int. J. Solids Structures, 46: 4192-4207. DOI: 10.1016/j.ijsolstr.2009.08.012.

Beldie, L., G. Sandberg and L. Sandberg, 2001. Paperboard packages exposed to static loads-finite element modeling and experiments. Packag. Technol. Sci., 14: 171-178.

Castro, J. and M. Ostoja-Starzewski, 2003. Elasto-plasticity of paper. Int. J. Plasticity, 19: 2093-2098. DOI: 10.1016/S0749-6419(03)00060-3.

Donaldson, G.R., 1954. An instrument for numerical measurement of bending uality of boards. Proceedings of the APPITA, pp: 237-250.

Halladay, J.F. and Ulm, R.W.K., 1939. Creasing and bending of folding boxboards. TAPPI Paper Trade J., 108-5: 36-40.

Hine, D.J., 1959. Testing boxboard creasing. Modern Packaging.

Isaksson, P. and R. Hägglund, 2005. A mechanical model of damage and delamination of corrugated board during folding. Eng. Fracture Mechani., 72: 2299-2315. DOI: 10.1016/j.engfracmech.2005.03.002

Isaksson, P., R. Hägglund and P. Gradin, 2004. Continuum damage mechanics applied to paper. Int. J. Solids Structures, 41: 4731-4755. DOI: 10.1016/j.ijsolstr.2004.02.043
Karafillis, A.P. and M.C. Boyce, 1993. A general anisotropic yield criterion using bounds and a transformation weighting tensor. J. Mechan. Phys. Solids, 41: 1859-1882.

Mäkelä, P. and S. Östlund, 2003. Orthotropic elastic-plastic material model for paper materials. Int. J. Solids Structures, 40: 5599-5620.

Nagasawa, S., Y. Fukuzawa, T. Yamaguchi, S. Tsukatani and I. Katayama, 2003. Effect of crease depth and crease deviation on folding deformation characteristics of coated paperboard. J. Mater. Proc. Technol., 140: 157-162. DOI: 10.1016/S0924-0136(03)00825-2

Nygårds, M., N. Hallbäck, M. Just and J. Tryding, 2005. A finite element model for simulations of creasing and folding of paperboard.

Sawyer, J.P.G., R. Jones and P.R. MacKinlay, 1998. A unified constitutive theory for paper. Composite Structures, 42: 93-100.

Stenberg, N., 2003. A model for the through-thickness elastic-plastic material behavior of paper. Int. J. Solids Structures, 40: 7483-7498. DOI: $10.1016 /$ j.ijsolstr.2003.09.003

Stenberg, N., 2002. On the out-of-plane mechanical behaviour of paper materials. PhD Thesis, Royal Institute of Technology (KTH).

Thakkar, B.K., L.G.J. Gooren, R.H.J. Peerlings and M.G.D. Geers, 2008. Experimental and numerical investigation of creasing in corrugated paperboard. Philosophical Magazine, 88: 3299-3310. DOI: $10.1080 / 14786430802342576$

Xia, Q.S., 2002. Mechanics of inelastic deformation and delamination of paperboard. PhD Thesis, Massachusetts Institute of Technology, USA.

Zakirov I., A. Nikitin, A. Naumov and N. Faizullina, 2015. Research of the process of shaping folded filler of sandwich panels from cardboard. Am. J. Applied Sci., 12: 938-944. DOI: 10.3844/ajassp.2015.938.944

Zakirov I.M. and I.I. Zakirov, 2014. Creasing on the elastic base. Russian Aeronautics, 4: 68-70

Zakirov I.M., A.V. Naumov and M.A. Semeshko, 2016. Shaping of polymer paper as filler for three-layer panels. Russian Eng. Res., 36: 201-205. 